Neurotoxicity towards the peripheral nervous system which appears clinically in the form of painful neuropathy is an essential dose-limiting factor during the treatment of multiple myeloma. In this review article different forms of this painful neuropathy are presented together with currently available diagnostic tools which are usually applied to confirm the diagnosis. Also, the most often used neurological scales estimating neurological deficit are presented. Special attention is paid to the management of the reversibility of bortezomib-induced neuropathic pain.

Key words: multiple myeloma, bortezomib, bortezomib induced neuropathy.

Contemp Oncol (Pozn) 2013; 17 (5): 421-426 DOI: $10.5114 /$ wo.2013.37214

\section{Bortezomib-induced painful neuropathy in patients with multiple myeloma}

\author{
Małgorzata Bilińska', Lidia Usnarska-Zubkiewicz², Anna Pokryszko-Dragan
}

1Department of Neurology, Wroclaw Medical University, Wroclaw, Poland

2Department of Haematology, Blood Neoplasms and Bone Marrow Transplantation, Wroclaw Medical University, Wroclaw, Poland

\section{Introduction}

There is growing evidence that introduction of bortezomib to the treatment of multiple myeloma (MM) not only results in longer survival but also, on the basis of comparative studies with different pharmacological regimens, enhances the percentage of MM patients with complete or partial responses [1-3]. One significant factor which limits treatment with bortezomib is its neurotoxicity, especially towards the peripheral nerves, that clinically appears in a form of painful sensory axonal neuropathy $[1,4-6]$. Contrary to subacute or chronic sensory paraneoplastic axonal neuropathies presenting with pain of medium intensity, abrupt onset and severe pain are the main features recorded during interview [7]. The occurrence of pain is strictly related to the time of introduction of bortezomib. Pain can be preceded by paraesthesia which subsequently is converted into pain. Painful neuropathy usually occurs within the first 5 cycles of treatment. When subsequent (more than 5) cycles are applied on a patient, there is only a slight tendency for enhancement in the incidence of neuropathy $[3,8,9]$. This supports the opinion that the dose effect of bortezomib is much more associated with the dose-threshold effect than with the cumulative-dose effect. The clinical and electrophysiological study performed by Stubblefied et al. pointed out that pre-existing damage involving the peripheral nervous system should also be considered as a risk factor in the susceptibility to bortezomib-induced neuropathy (BIN) in MM patients [10].

\section{Neurophysiology/neuropathology of bortezomib-induced neuropathy}

Sensory impulses from the periphery are conveyed into the central nervous system. Each specific sensory modality (superficial sensation such as touch, pain, and temperature, and deep sensation such as vibration or position sense) has its own receptors and triggers impulse of determined modality transmitted along nerve fibres [11-14]. After approaching the spinal cord superficial and deep impulses are conveyed through separated pathways centrally into the cortex. Some impulses (part of deep sensation) which run into the subcortical structures are utilized to maintain the proper posture, balance, gait, and smoothness of movements. Monitoring of sensory perception can be carried out only among conscious human subjects. Simultaneous assessment of the activity of the fibres involved in superficial sensory function in sensory cutaneous nerves (recorded in the form of evoked potentials on neurograms) and human's perception of sensation can be performed using the cutaneous nerve block procedure. In this method a blocking procedure, such as increasing pressure applied to the skin supplied by the examined nerve, triggers changes in neurograms in response to standardized electrical stimulus given to this nerve. Simultaneously, a person who has undergone this blocking procedure determines changes in sensation in response to the standardized electrical stimulus with increasing 
pressure applied to the skin. It should be emphasized that only those impulses which reach the cortex and which are subsequently encoded and analyzed in the higher centres of the cortex are available to be perceived [15].

Morphologically, sensory fibres differs in diameter. Some of them are covered with myelin while others are unmyelinated. Superficial sensation is transmitted along both unmyelinated fibres (C), which are mainly responsible for pain sensation, and also along thin myelinated ones (A $\delta$ ) which propagate thermal and light touch sensation [12, 13]. Deep sensation is conducted by the large myelinated sensory ones (A $\beta$ ) [14]. In BIN all types of afferent fibres are affected with a preponderance of unmyelinated and thin myelinated fibres. This results in pain with or without superficial sensory deficit on neurological examination.

\section{Pathogenesis of bortezomib-induced neuropathy}

The mechanisms involved in the toxic action against peripheral nerves are not fully understood. On the basis of experimental studies it has been hypothesized that damage in patients treated with bortezomib occurs in the dorsal root ganglia (DRG) neurons (especially satellite ones) and Schwann cells [16]. Mitochondria and endoplasmic reticulum are the structures injured in cells. The blood-nerve barrier at the level of DRG does not strictly separate these two compartments, which allows toxic bortezomib to penetrate into the nerve compartment, which is not observed along nerve fibres in the nerve's trunk. A toxic agent may disturb the oxidative phosphorylation in mitochondria with subsequent dysregulation of calcium homeostasis [16]. Dysregulation of neurotrophins is also taken into consideration. It has been demonstrated that bortezomib inhibits the activity of the transcription factor nuclear factor $\kappa \mathrm{B}(\mathrm{NF}-\kappa \mathrm{B})$ [17]. This action results in blockade of transcription of nerve growth factor-mediated neuron survival. Another possibility is that bortezomib's neuropathy is associated with immune-mediated mechanisms [18]. This etiology is considered in cases with clinical findings resembling those typical for polyradiculoneuropathy. Suspicion of immune etiology in those cases is strengthened by a positive response to anti-inflammatory treatment [18].

\section{Clinical findings in bortezomib-induced neuropathy}

The assessment of BIN is based mainly on an interview and neurological examination. Symptoms described by affected patients are characterized by neuropathic pain. Pain is denominated as burning, shooting, electric-like feeling, drilling, and/or stabbing [19]. At the beginning paraesthesia can be prickling in character. The other subjective descriptors connected with unpleasant feeling include so-called "negative" sensory symptoms such as numbness, tightness, and coldness [20]. On neurological examination concerning function of the sensory system, sensory loss to all modalities of superficial sensation is distal, symmetrical in distribution and length-dependent $[19,20]$. Length-dependent pattern appears when the primary damage is localized to the cell soma of neurons. It disrupts structures such as mito- chondria, which results in lack of energy. This limits protein transporters' cargo of neurotransmitters, ions and other substances along the microtubules to the distal parts of axons. The most distal parts of the longest axons are affected first, so clinically symptoms appear first in the foot and then in fingertips. Symptoms are later experienced in more proximal parts of extremities. Damage to $A \beta$ fibres is less pronounced in comparison with the injury of $A \delta$ and $C$ ones. If present it occurs in abnormal proprioception. Abnormalities in two-point discrimination, astereognosis, can appear. Patients complain of walking disturbances (walking on stones/crumpled socks); there is also positive Romberg sign. Tendon deep reflexes and muscle strength are always normal in painful sensory neuropathies. They are affected only in case of $A \beta$ fibres involvement. Rarely the BIN can clinically appear as ganglionopathy [21, 22]. This unit is characterized by loss of sensory neurons in DRG which causes the degeneration of short and long peripheral axons and central sensory projections in the posterior columns. The pathological process leads to a pattern of sensory nerve damage that is not length-dependent. Instead, this pattern is patchy in distribution and affects asymmetrically primarily proximal dermatomes, innervated by shorter axons. Both superficial and deep sensation are abnormal. On examination sensory deficit involves dermatomes of face, arms and trunk, and later can be found on distal parts of limbs. There is also autonomic impairment in patients with BIN. Preferential damage occurs in cholinergic and skin vasomotor fibres, resulting mainly in gastrointestinal and sweating disturbances, and also in skin discoloration and slight oedema [1, 2]. Orthostatic hypotension and cardiac arrhythmias are not typical for small-fibre sensory neuropathies. Later in the course of neuropathy with the progressive involvement of $A \beta$ fibres gait unsteadiness may progress to overt ataxia. In BIN, in cases of not clinically overt disturbances in deep sensation, damage to the $A \beta$ fibres can be revealed by neurophysiological methods [23].

\section{Diagnostic procedures in monitoring of bortezomib-induced neuropathy}

Detailed interview together with clinical examination, with special attention paid to the presence of symptoms and signs of small-fibre neuropathy, is the first step in the diagnosis of BIN. The other methods, especially neurophysiological ones, should also be introduced to strengthen the diagnosis. Standard neurographic sensory nerve studies are of little significance since the damage to small calibre sensory afferents did not reveal any changes in neurographic parameters. When changes involve large calibre sensory afferents (A $\beta$ fibres), that is there is an axonal large-fibre sensory neuropathy, the lowering or lack of the amplitude of sensory nerve action potential (SNAP) is expected with no or little changes in conduction velocity along the examined nerve. Axonal small-fibre neuropathy does not affect either the SNAP parameters or sensory velocity. Recently, quantitative sensory testing (QST) - a new neurophysiological assessment of function of sensory afferents - has been introduced in evaluating small-fibre sensation (A $\delta$ and C fibres) [24]. This method evaluates thermal (warm, cold) and pain (cold pain, heat pain) 
thresholds in selected dermatomes. Quantitative sensory testing is simple in use, time-saving, but it needs good cooperation between an examiner and a patient. It should be stressed that abnormal thresholds can result from the damage located anywhere along the pathway between dermatomes and cortex, so to establish the proper diagnosis the QST results ought to be considered together with clinical data. BIN as a preferentially small-fibre neuropathy can be evaluated immunohistochemistry with the intra-epidermal nerve fibre density (IENFD) method [19]. As it is known, epidermal nerves are the distal terminals of small DRG neurons that cross the epidermal basement to innervate the epidermis. These axons' terminals are visualized by interaction of antibodies to neuropeptide protein gene product GPG 9.5 (a ubiquitin hydrolase), the panaxonal marker. Immunofluorescent staining assesses the density of nerve terminals in skin biopsy. Among healthy subjects, the proximal-distal gradient in density has been established. It means that there is higher density in IENFD in proximal sites of limbs in comparison with the distal ones [25]. In the case of distal, symmetrical small-fibre neuropathy this gradient is expected to be higher than in healthy controls. In bortezomib-induced ganglionopathy the gradient of extremities is the same as in healthy subjects [25]. Neurophysiological assessment of deep sensation is available by examination of the vibratory threshold with Vibratory System Testing. Pain and other sensory disturbances experienced by patients depend on different variables such as personality (mainly temperament) and resilience. Resilience from the psychological point of view denotes the effective ability to cope with and overcome adversities. Both personality and resilience are inherited features, so the degree of experienced pain and unpleasant sensation differs among people. Nevertheless, toxicity to peripheral nerves leads to limitation of daily activities and more profoundly diminishes the Quality of Life. Finally, treatment of MM can be discontinued because of adverse events. In order to trace the progression of disability in the course of neuropathy secondary to chemotherapy different grading scales have been introduced. The most widely used is the National Cancer Institute-Common Toxicity Criteria (NCI-CTC) grading scale [26]. Depending on the type of the affected fibres in the nerve trunk the sensory or motor scales have been applied. The sensory scale ( $\mathrm{SNCl-CTC)}$ describes the impact of sensory disturbances on a wide range of everyday functions and also indicates to what extent these disturbances interfere with daily living activities (DLA). The higher the grade, the higher is the level of disability. Grading in this scale is as follows:

- grade 1 - asymptomatic, loss of deep reflexes or paraesthesia (including tingling) but not interfering with function, - grade 2 - sensory alteration or paraesthesia (including tingling) interfering with function but not with DLA,

- grade 3 - sensory alteration or paraesthesia interfering with DLA,

- grade 4 - disabling,

- death.

The other scale which has been used to monitor painful $\mathrm{BIN}$ is the FACT/GOG-Ntx subscale (Functional Assessment of Cancer Therapy scale/Gynecologic Oncology Group-Neurotoxicity) [26]. This self-reported questionnaire is used to determined both the patient's quality of life (27 questions concerning physical, social, emotional and functional patient's well-being) and the impact of neurotoxicity on activity impairment (11 questions). Each answer is graded from 0 (not at all) to $4-$ a distinctly experienced disability (very much). The examination with the FACT/GOG-Ntx questionnaire in the group of MM patients just prior to introduction of bortezomib into therapy revealed a correlation between a high score on this scale and the development of severe neuropathy in the course of bortezomib treatment [9]. Total neuropathy score (TNS) has also been introduced into the evaluation of BIN $[26,27]$. Total neuropathy score comprises information concerning sensory, motor, and autonomic symptoms, selected data (clinical signs) which may be abnormal in sensory painful neuropathy such as pin sensation, vibration sensibility, strength, and tendon reflexes. Moreover, data obtained from electrophysiological examinations such as the amplitude of the SNAP of the sural nerve, and the amplitude of the compound muscle action potential (CMAP) of the peroneal nerve as well as QST Vibration Sensation are also included in TNS.

In both clinical trials "Summit" and "Crest" in which patients with relapsed/refractory MM were treated with bortezomib the occurrence of BIN was reported to be as high as 35\% [9]. Results of both these trials also demonstrated that the degree of sensory nerve dysfunction according to sNCI-CTC v.2.0 and the percentage of MM patients with overt neuropathy were dose-dependent. In the first group of MM patients who were treated with a lower dose $\left(1 \mathrm{mg} / \mathrm{m}^{2}\right)$ overt neuropathy was detected in $21 \%$, while in the second group, treated with a higher dose $\left(1.3 \mathrm{mg} / \mathrm{m}^{2}\right)$, the proportion of MM patients with neuropathy was as high as $37 \%$. Moreover, when compared with the second group (81\%), the incidence of neuropathy with grade 2 and 3 according to $\mathrm{NCl}-\mathrm{CTC}$ was lower in the first group (31\%). In the phase III Apex trial MM relapsed patients were included with neuropathy not exceeding grade 1 according to sNCI-CTC v.2.0 [28]. They were treated with bortezomib at dosage $1.3 \mathrm{mg} / \mathrm{m}^{2}$. Treatment-induced neuropathy occurred in 37\% of patients, grade 2 or higher was found in $27 \%$ of them, grade 3 and grade 4 was assessed in $9 \%$ and less than 1\% of treated patients, respectively. Although different treatment schedules used in MM patients prior to bortezomib therapy can potentiate bortezomib-induced neuropathy, it should be stated that the MM patients with the initial bortezomib treatment are also at risk of neuropathy. Richardson et al. in 2009 presented data concerning the incidence and the severity of bortezomib-induced neuropathy in newly diagnosed MM patients [1]. Assessment was performed according to SNCl-CTC v. 3.0., and bortezomib was administered at the dose of $1.3 \mathrm{mg} / \mathrm{m}^{2}$ per cycle. Prior to therapy neuropathy was discovered in $20 \%$ of patients (grade 1 in $18 \%$, and grade 2 in $2 \%$ of patients). At the end of the treatment peripheral nerve palsy was confirmed in $64 \%$ of them (grade 1, 2, and 3 in 36\%, 25\%, and 3\%, respectively).

\section{Therapeutic standards}

Painful sensory neuropathy can develop at any time of bortezomib therapy but the onset of sensory disturbances usually takes place at the fifth cycle at cumulative dosage equal to $30 \mathrm{mg} / \mathrm{m}^{2}$. The risk of occurrence of neuropathy of 
severe course, e.g. with grade 3 or higher according to $\mathrm{sNCl}$ CTC, has been increasing slightly up to the eighth cycle and thereafter it has revealed a tendency to keep a stable level $[2,3]$. It has been suggested that neurotoxicity towards peripheral sensory nerves is related to the threshold-dose rather than to the cumulative-dose. It has also been verified that dose-modification schedules for treatment with bortezomib adjusted to the severity of clinically overt sensory neuropathy are able to reverse the neurological deficit. Richardson et al. investigated the impact of bortezomib dose reduction or discontinuation in MM patients with treatment-emergent neuropathy. Thirty-seven percent (91/331) of patients examined by the cited authors presented sensory neuropathy grade 2 or higher [28]. Dose-modification procedures were highly effective and resulted in significant improvement in 64\% (58/91) of the patients. This significant improvement included resolution of neuropathy to the level as it was prior to the bortezomib therapy (50 patients) or decrease by at least one grade on the $\mathrm{sNCl}-\mathrm{CTC}$ scale (8 patients). Patients who have achieved such a good result came from 3 groups of different types of dose modification (A, B, and C). In group A (grade no more than 2) with no modification of treatment a significant improvement to the level just prior to the treatment was achieved in 9 out of 19 patients in a median of 106 days. Group B consisted of 41 MM patients with neuropathy. Dose reduction or temporary discontinuation of bortezomib was made. In 71\% (29/41) of patients resolution of neuropathy was achieved in a median of 78 days. In group C, permanent discontinuation was performed. In this group improvement or resolution of sensory nerve palsy was found in 20 patients (65\%) in a median of 122 days. The obtained results demonstrate that the percentage of improvements depends on a strategy applied to counteract sensory nerve injury. A higher proportion of improvement has been achieved with dose-modification schedules (74\% of the affected persons) in comparison with the treatment with no dose-modification strategy (improvement only in $47 \%$ of the affected patients). Dose-modification regimens due to clinical appearance of BIN are presented in Table 1 [according to 9].

Neuropathic pain negatively influences all aspects of DLA and profoundly lowers quality of life. In BIN the primary site of attack includes DRG neurons with subsequent sensory fibre involvement as it is supposed to be in other chemotherapyinduced neuropathies. Earlier, different pharmaceutical regimens were given to $\mathrm{MM}$ patients, and prior to the introduction of bortezomib therapy into the treatment of MM, thalidomide (of high neurotoxicity towards sensory nerves) was widely used. It has been speculated that successive addi- tion of the new treatment to the old one might potentiate the level of experienced pain. Data concerning the impact of the previous treatment with thalidomide on pain during bortezomib treatment differs. According to Badros et al. the severity and incidence of BIN depends on the presence of previously existing thalidomide neuropathy [6]. Similarly, Lanzani et al. underline the relationship between previously given thalidomide and risk level of BIN [27]. Contrary to the cited authors, Richardson et al. and Cata et al. did not find any impact of previous thalidomide treatment in MM patients and $\operatorname{BIN}[9,23,28]$. The impact of previously used chemotherapy regimens on the development of neuropathic pain during the treatment with bortezomib was also analyzed by Corso et al. [5]. Cited authors subdivided MM patients treated with bortezomib into two groups. The first one comprised MM patients who had not been treated, while the second one comprised patients who had been given chemotherapy regimens. Polyneuropathy developed on average within the $4^{\text {th }}$ cycle with the same incidence in both groups (about 50\% affected in each group). Neuropathic pain, however, was much more often recorded in the group which was previously treated with chemotherapy (81\%) than in the group which was not subjected to earlier treatment (50\%). After the modification of treatment took place, neuropathic pain disappeared faster in the group without previous treatment (median time to pain resolution was 35 and 95 days, respectively).

There have been many pharmacological studies assessing alleviation of neuropathic pain in neuropathies of etiology different than CIPN, mostly in patients with postherpetic neuralgia or in patients with painful diabetic neuropathy [2932]. On the basis of the results of these studies the first and the second line of therapies in the treatment of neuropathic pain in studied diseases are recommended [33-35]. They can be added to dose-modification schedules of bortezomib, but it is worth remembering that patients with sensory neuropathy induced by bortezomib could be resistant to them. The choice of medication in an individual case may depend on many factors. Among them potential for adverse events (AEs), drug interactions, and risk of abuse have to be taken into consideration. In general, data concerning safety, efficacy and tolerability of pain relief therapy have to be recognized with caution. It should be a rule to introduce small doses with subsequent slow titration to effective dosage. In case of an AE the therapy needs to be discontinued. Some neuropathic patients treated with one class of pain relief medication could respond while other patients could fail to respond. A partial response to selected first-line therapy can be changed to a complete response by adding another first-

Table 1. Modification of bortezomib dosage in relation to the severity of painful neuropathy assessed by the sNCI-CTC scale

\begin{tabular}{ll} 
SNCI-CTC scale & Dosage of bortezomib \\
\hline Grade 1 - paresthesias, loss of reflexes, function prevented, lack of pain & no action undertaken \\
\hline Grade 1 with pain or grade 2 (function difficulties without influence on ADL) & dose should be reduced to $1 \mathrm{mg} / \mathrm{m}^{2}$ \\
\hline Grade 2 with pain or grade 3 (influence on ADL) & $\begin{array}{l}\text { withhold treatment until neurotoxicity resolution takes place } \\
\text { with subsequent introduction of bortezomib at lower dose } \\
\left(0.7 \mathrm{mg} / \mathrm{m}^{2} \text { once weekly) }\right.\end{array}$ \\
Grade 4 (permanent severe sensory dysfunction) & permanent discontinuation of treatment with bortezomib
\end{tabular}


line therapy (combination therapy). Nevertheless, the disadvantage of combination therapy comes from an increased risk of AEs. The data concerning the period within which the pain relief treatment should be maintained at a stable level is difficult to establish. It lasts 4 to 6 weeks, and thereafter slow lowering of dosage is preferred.

First-line therapies:

1. Calcium channels $\alpha 2-\delta$ ligands (gabapentin and pregabalin). Both bind to voltage-gated calcium channels at the $\alpha 2-\beta$ subunit and inhibit neurotransmitter release. Gabapentin should be initiated at low doses (100-300 mg) 3 times a day with gradual increase until pain relief or dose-limiting AE. The increase by 100-300 mg 3 times daily should be performed every 7 days. Maximal dose is as high as $3600 \mathrm{mg}$ per day. Pregabalin - beginning dosage has been established as $50 \mathrm{mg} 3$ times daily or $75 \mathrm{mg}$ twice daily. Titration to effective dose which on average equals $300 \mathrm{mg} /$ day can be achieved within 1 or 2 weeks. Both have been used as antiepileptic drugs, but they also possess high pain relief potential. Renal impairment needs dosage modification (in relation to creatinine clearance). Side effects such as daytime sedation, somnolence, dizziness, gait ataxia, and gastrointestinal symptoms are mostly observed.

2. Selective serotonin and norepinephrine reuptake inhibitors (SSNRIS). Duloxetine - initial dose $30 \mathrm{mg}$ per day should be increased to $60 \mathrm{mg}$ within a week. Venlafaxine - starting dosage $37.5 \mathrm{mg}$ twice a day ought to be increased to efficacious dosage (150-225 mg) within 2 to 4 weeks or longer. Side effects of SSNRIs include dizziness, somnolence, nausea, and constipation. Venlafaxine can rarely trigger cardiac arrhythmias and arterial blood hypertension.

3. Tricyclic antidepressants (TCAs). This class of medications is very effective in alleviating pain syndromes. Nevertheless, numerous AEs limit their usage. Because of cardiotoxicity and the negative impact on cognitive functions they should not be recommended to older patients. They are contraindicated in glaucoma (increase in intraocular hypertension). They exert anticholinergic side effects and also trigger psychotic episodes. Nortriptyline should be initiated at a low dose of 10 or 25 mg once a day in late evening. The dose, if necessary, may be increased by 10 or $25 \mathrm{mg}$ every 7 days. The effective dose (if tolerated) is as high as $100 \mathrm{mg}$ daily. Amitriptyline - the initial dosage and titration are the same as with nortriptyline. AEs are much more severe and appear with higher incidence in comparison with AEs of nortriptyline.

4. Topical lidocaine. The 5\% lidocaine patches are recommended especially in well-localized neuropathic pain. No more than 3 patches daily should be adhered to the painful region for no more than 12 hours. There are no data indicating that more patches applied to the skin give better pain relief. Treatment lasts for 3 weeks.

5. Opioid analgesics. Recently, this class of first line-therapy has not been recommended for the treatment of neuropathic pain unless the other first-line regimens are effective. Long-term opioid therapy develops physical and psychological dependence. Always sedation, constipation and nausea accompany. Initial doses should be low; gradual titrating and reduction needs time. For pain relief, tra- madol has been used extensively. It is a weak $\mu$-opioid receptor agonist which also has the potential to inhibit reuptake of serotonin and norepinephrine. Initial dosage should be as low as 50 mg once or twice a day. Titration up to $400 \mathrm{mg}$ daily can be achieved by increase of the daily dose by 50 to 100 mg every 3 to 7 days. In older patients, at age 70 or more with renal and hepatic damage lower dosage up to $300 \mathrm{mg}$ per day is recommended.

In summary the authors emphasize that despite the high risk of BIN currently introduced algorithms of dose-limiting schedules have been successful in pain relief. This allows therapy to be continued, which results in a longer life span without deterioration of daily living for MM patients. It is also of significance that contrary to the previously used regimens in plasmacytic dyscrasias including alkylating agents, among them melphalan, bortezomib therapy does not increase the risk for developing secondary neoplasms [36].

\section{The authors declare no conflict of interests.}

\section{References}

1. Richardson PG, Xie W, Mitsiades C, et al. Single-agent bortezomib in previously untreated multiple myeloma. Efficacy, characterization of peripheral neuropathy, and molecular correlations with response and neuropathy. I Clin Oncol 2009; 27: 3518-25.

2. Richardson PG, Barlogie B, Berenson J, et al. A phase 2 study of bortezomib in relapsed, refractory myeloma. N Engl J Med 2003; 348: 2609-17.

3. Jagannath S, Barlogie B, Berenson J, et al. A phase 2 study of two doses of bortezomib in relapsed or refractory myeloma. Br J Haematol 2004; 127: 165-72.

4. Walter-Croneck A, Dmoszyńska A, Skotnicki A, et al. Skuteczność i bezpieczeństwo bortezomibu w leczeniu opornego/nawrotowego szpiczaka plazmocytowego - doświadczenia Polskiej Grupy Szpiczakowej. Acta Haematol Pol 2006; 37 Suppl. 1: 125-34.

5. Corso A, Mangiacavalli S, Varettoni M, Pascutto C, Zappasodi P, Lazzarino M. Bortezomib-induced peripheral neuropathy in multiple myeloma: a comparison between previously treated and untreated patients. Leuk Res 2010; 34: 471-4.

6. Badros A, Goloubeva O, Dalal JS, et al. Neurotoxicity of bortezomib therapy in multiple myeloma: a single-center experience and review of the literature. Cancer 2007; 110: 1042-8.

7. Oki Y, Koike H, lijima M, et al. Ataxic vs painful form of paraneoplastic neuropathy. Neurology 2007; 69: 564-72.

8. San Miguel JF, Schlag R, Khuageva NK, et al. Bortezomib plus melphalan and prednisone for initial treatment of multiple myeloma. N Engl J Med 2008; 359: 906-16.

9. Richardson PG, Briemberg H, Jagannath S, et al. Frequency, characteristic, and reversibility of peripheral neuropathy during treatment of advanced multiple myeloma with bortezomib. J Clin Oncol 2006; 24: 3113-20.

10. Stubblefield MD, Slovin S, MacGregor-Cortelli B, et al. An electrodiagnostic evaluation of the effects of pre-existing peripheral nervous system disorders in the patients treated with novel proteasome inhibitor bortezomib. Clin Oncol 2006; 18: 410-8.

11. Campero M, Serra J, Ochoa JL C-polymodal nociceptors activated by noxious low temperature in human skin. J Physiol 1996; 497: 565-72.

12. Chen CC, Rainville P, Bushnell MC. Noxious and innocuous cold discrimination in humans: evidence of separate afferent channels. Pain 1996; 68: 33-43

13. Ochoa J, Torebjörk E. Sensations evoked by intraneural microstimulation of C nociceptor fibres in human skin nerves. J Physiol 1989; 415: 583-99. 
14. Vallbo AB, Johansson RS. Properties of the cutaneous mechanoreceptors in the human hand related to touch sensation. Hum Neu robiol 1984; 3: 3-14.

15. Dobrogowski J, Zajączkowska R, Dutka J, Wordliczek J. Patofizjologia i klasyfikacja bólu. Pol Przegl Neurol 2011; 7: 20-30.

16. Meregalli C, Canta A, Carozzi VA, et al. Bortezomib-induced painfu neuropathy in rats: a behavioural, neurophysiological and pathological studies in rats. Eur J Pain 2010; 14: 343-50.

17. Lenz HJ. Clinical update: proteasome inhibitors in solid tumors. Can cer Treat Rev 2003; 29: 41-8.

18. Ravaglia S, Corso A, Piccolo G, et al. Immune-mediated neuro pathies in myeloma patients treated with bortezomib. Clin Neurophysiol 2008; 119: 2507-12

19. Holland NR, Crawford TO, Hauer P, Cornblath DR, Griffin JW, McArthur JC. Small-fiber sensory neuropathies: clinical course and neuropathology of idiopathic cases. Ann Neurol 1998; 44: 47-59.

20. Hoitsma E, Reulen JP, de Baets M, Drent M, Spaans F, Faber CG. Small fiber neuropathy: a common and important clinical disorder. J Neurol Sci 2004; 227: 119-30.

21. Sghirlanzoni A, Pareyson D, Lauria G. Sensory neuron disease. Lancet Neurol 2005; 4: 349-61.

22. Khan S, Zhou L. Characterization of non-length-dependent smallfiber sensory neuropathy. Muscle Nerve 2012; 45: 86-91.

23. Cata JP, Weng HR, Burton AW, Villareal H, Giralt S, Dougherty PM. Quantitative sensory findings in patients with bortezomib-induced pain. J Pain 2007; 8: 296-306.

24. Rolke R, Magerl W, Campbell KA, Schalber C, Caspari S, Birklein F, Treede RD. Quantitative sensory testing: a comprehensive protocol for clinical trials. Eur J Pain 2006; 10: 77-88.

25. Lauria G, Sghirlanzoni A, Lombardi R, Pareyson D. Epidermal nerve fiber density in sensory ganglionopathies: clinical and neuro physiologic correlations. Muscle Nerve 2001; 24: 1034-9.

26. Cavaletti G, Frigeni B, Lanzani F, Mattavelli L, Susani E, Alberti P, Cortinovis D, Bidoli P. Chemotherapy-induced peripheral neurotoxicity assessment: a critical revision of the currently available to tools. Eur J Cancer 2010; 46: 479-94.

27. Lanzani F, Mattavelli L, Frigeni B, Rossini F, Cammarota S, Petrò D, Jann S, Cavaletti G. Role of pre-existing neuropathy on the course of bortezomib-induced peripheral neurotoxicity. J Peripher Nerv Syst 2008; 13: 267-74.

28. Richardson PG, Sonneveld P, Schuster MW, et al. Reversibility of symptomatic peripheral neuropathy with bortezomib in the phase III Apex trial in relapsed multiple myeloma: impact of dose modification guideline. Br J Haematol 2009; 144: 895-903.

29. Backonja M, Beydoun A, Edwards KR, Schwartz SL, Fonseca V, Hes M, LaMoreaux L, Garofalo E. Gabapentin in the symptomatic treatment of painful neuropathy in patients with diabetes mellitus. JAMA 1998; 280: 1831-6

30. Rosenstock J, Tuchman M, LaMoreaux L, Sharma U. Pregabalin for treatment of painful diabetic peripheral neuropathy: a double-blind placebo-controlled trials. Pain 2004; 110: 628-38.

31. Harati Y, Gooch C, Swenson M, et al. Double-blind randomized trial of tramadol for the treatment of the pain of diabetic neuropathy. Neurology 1998; 50: 1842-6.

32. Goldstein DJ, Lu Y, Detke MJ, Lee TC, Iyengar S. Duloxetine vs. placebo in patients with painful diabetic neuropathy. Pain 2005; 116 109-18.

33. Gidal BE. New and emerging treatment options for neuropathic pain. Am J Manag Care 2006; 12 (9 Suppl): S269-S78.

34. Dworkin RH, O'Connor AB, Audette J, et al. Recommendations for the pharmacological management of neuropathic pain: an overview and literature update. Mayo Clin Proc 2010; 85 (3 Suppl): S3-S14.

35. Wordliczek J, Zajączkowska R, Dobrogowski J. Farmakologiczne leczenie bólu neuropatycznego. Pol Przegl Neurol 2011; 7: 39-48.

36. Piszcz J, Bolkun L, Cichocka E, Kłoczko J. Secondary acute lym phoblastic leukemia in a multiple myeloma patient. Wspolczesna Onkol 2012; 16: 593-5.

\section{Address for correspondence}

Małgorzata Bilińska MD, PhD

Bacciarellego 35B/3

51-649 Wrocław

e-mail: mbilinsk@mp.pl

Submitted: 16.11 .2012

Accepted: $\quad 9.04 .2013$ 